\title{
Use of Advance Care Planning Billing Codes for Hospitalized Older Adults at High Risk of Dying: A National Observational Study
}

\author{
Amber E Barnato, MD, MPH, MS ${ }^{1 *}$; A James O'Malley, PhD 1,2; Jonathan S Skinner, PhD 1,3; John D Birkmeyer, MD ${ }^{1,4}$
}

\begin{abstract}
${ }^{1}$ The Dartmouth Institute for Health Policy and Clinical Practice, Geisel School of Medicine at Dartmouth, Lebanon, New Hampshire; ${ }^{2}$ Department of Biomedical Data Science, Geisel School of Medicine at Dartmouth, Lebanon, New Hampshire; ${ }^{3}$ Department of Economics, Dartmouth College, Hanover New Hampshire; ${ }^{4}$ Sound Physicians, Tacoma, Washington.
\end{abstract}

We analyzed advance care planning (ACP) billing for adults aged 65 years or above and who were managed by a large national physician practice that employs acute care providers in hospital medicine, emergency medicine and critical care between January 1, 2017 and March 31, 2017. Prompting hospitalists to answer the validated "surprise question" (SO; "Would you be surprised if the patient died in the next year?") for inpatient admissions served to prime hospitalists and triggered an icon next to the patient's name. Among 113,621 hospital-based encounters, only 6,146 (5.4\%) involved a billed ACP conversation: $8.3 \%$ among SQ-prompted who answered "no" and $4.1 \%$ SQ-prompted who answered "yes" (for non-SO prompted cases, the fraction was $3.5 \% ; P<.0001)$. ACP conversations were associated with a comfort-focused care trajectory. Low ACP rates among even those with high hospitalistpredicted mortality risk underscore the need for quality improvement interventions to increase hospital-based ACP. Journal of Hospital Medicine 2019;14:229-231. (c) 2019 Society of Hospital Medicine

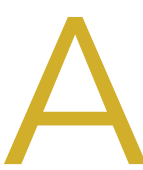

dvance care planning (ACP) is the process wherein patients, in discussions with their healthcare providers, family members, and other loved ones, make individual decisions about their future healthcare or prepare proxies to guide future medical treatment decisions. ${ }^{1,2}$ In 2016, the Centers for Medicare and Medicaid Services (CMS) began paying providers for ACP by using billing codes 99497 (first $30 \mathrm{~min}$ of ACP) and 99498 (additional $30 \mathrm{~min}$ of ACP). According to the CMS, during the first year after the billing codes were introduced, 22,864 providers billed for ACP conversations with 574,621 patients. ${ }^{3}$ While all adults are eligible, common triggers for ACP include advanced age, serious illness, and functional status changes that confer an increased risk of dying. We explored the early uptake of the ACP billing code in a large national physician practice that provided mandatory education in use of the ACP billing code, offered a small financial incentive for ACP documentation, and primed physicians to reflect on the patient's risk of dying in the next year at the time of hospital admission.

\section{METHODS}

We analyzed ACP billing for hospitalized adults aged 65 years or above and who were managed by a large national physician practice that employs acute care providers in hospital medi-

\footnotetext{
*Corresponding Author: Amber E. Barnato, MD, MPH; E-mail: amber.barna-
} to@dartmouth.edu; Telephone: 650-653-0829; Twitter: @abarnato

Received: August 31, 2018; Revised: December 7, 2018;

Accepted: December 20, 2018

๑ 2019 Society of Hospital Medicine DOI 10.12788/jhm.3150 cine, emergency medicine and critical care between January 1, 2017 and March 31, 2017. This practice employs approximately 2,500 hospital-based physicians in 250 community hospitals in 38 states. They collect data through handheld and desktop information technology (IT) tools to facilitate coding, billing, and compliance by hospitalists. Hospitalists receive mandatory web-based training in compliance with CMS ACP billing and templated ACP documentation. Additionally, they receive web-based training in serious illness communication skills during the first two years of employment. The training includes didactic content regarding steps for collaborative decision making, words to use during the encounter, and videos of simulated patient encounters demonstrating best practices. Hospitalists also receive a small financial incentive (\$20) for each properly documented ACP conversation that meets CMS criteria for ACP code payment.

Beginning in 2017, hospitalists were required to answer the validated Surprise Question (SQ; "Would you be surprised if the patient died in the next year?") for all admitted patients aged 65 years and older. The SQ is useful because it is intuitive and not burdensome for physicians to answer. Moreover, it is predictive of mortality. The pooled prognostic characteristics of the $\mathrm{SQ}$ across multiple populations for predicting the outcome of death at 6 months to 18 months include a sensitivity of $67.0 \%$ (95\% confidence interval [Cl] 55.7\%-76.7\%), a specificity of $80.2 \%$ (95\% Cl 73.3\%-85.6\%), a positive likelihood ratio of $3.4(95 \% \mathrm{Cl} 2.8-4.1)$, a negative likelihood ratio of 0.41 (95\% $\mathrm{Cl} 0.32-0.54)$, a positive predictive value of $37.1 \%(95 \% \mathrm{Cl}$ $30.2 \%-44.6 \%)$, and a negative predictive value of $93.1 \%(95 \%$ Cl 91.0\%-94.8\%). ${ }^{5}$ The SQ primed the admitting physician and triggered an "EoL" (end-of-life) icon next to the patient's name on the hospitalists' handheld electronic patient census. 
TABLE 1. Advance Care Planning Billing by Response to the "Surprise Question"

\begin{tabular}{|c|c|c|}
\hline & $\begin{array}{l}\text { ACP billed } \\
(n=6,146)\end{array}$ & $\begin{array}{l}\text { No ACP billed } \\
(n=107,466)\end{array}$ \\
\hline $\begin{array}{l}\text { "No, I would not be surprised if the patient died in } \\
\text { the next year" }(n=41,276)\end{array}$ & $3,414(8.3 \%)$ & $37,862(91.7 \%)$ \\
\hline $\begin{array}{l}\text { "Yes, I would be surprised if the patient died } \\
\text { in the next year" } \\
(n=32,441)\end{array}$ & $1,317(4.1 \%)$ & $31,124(95.9 \%)$ \\
\hline $\begin{array}{l}\text { Not prompted to answer SQ } \\
(n=39,881)\end{array}$ & $1,414(3.5 \%)$ & $38,467(96.5 \%)$ \\
\hline \multicolumn{3}{|c|}{$\begin{array}{l}\text { aThe SQ was not asked of all patients in the first quarter of } 2017 \text {. This included hospitaliza- } \\
\text { tions at sites that had delayed implementation of the SQ during the quarter and all patients } \\
\text { who were on observation status }\end{array}$} \\
\hline Abbreviations: $\mathrm{ACP}$, advance care planning; $\mathrm{SQ}, \mathrm{s}$ & prise question. & \\
\hline
\end{tabular}

We summarized ACP billing rates and used mixed-effects regression to estimate adjusted ACP rates accounting for patient covariates and clustering at the provider and hospital level. Patient covariates included age; answer to the SO ["yes," "no," or "missing"]); and the presence or absence of seven comorbidities: dementia, heart failure, chronic obstructive pulmonary disease, renal failure, liver failure, metastatic cancer, and nonmetastatic cancer. We quantified the magnitude of provider and hospital variation in ACP rates by using the intraclass correlation coefficient (ICC).

\section{RESULTS}

In the first quarter of 2017, hospitalists admitted 113,612 patients aged 65 years and older. Hospitalists were prompted to answer the SQ for 73,731 (65\%) of the patients. They were not prompted to answer the SQ for 39,881 (35\%) of the patients (ie, missing data for the SQ). Reasons for not prompting include delayed implementation at a site and the patient not being admitted to the hospital (eg, managed on observation status). When prompted, hospitalists answered "no" to the SO for $41,276 / 73,731(56 \%)$ of admissions.

Only 6,146/113,612 (5.4\%) of all admissions involved a billed ACP conversation. Rates were highest among SQ-prompted/ answer "no" cases (8.3\%) compared with SQ-prompted/answer "yes" cases (4.1\%) and non-SO-prompted cases (3.5\%), with all pairwise differences being statistically significant $(P$ values "yes" vs "no" $=.0079$, "yes" vs not prompted $=.0043$, "no" vs not prompted <.0001; see Table 1).

In addition to being more likely to have a "no" response to the $\mathrm{SQ}$, those with a billed ACP conversations were older (80 vs $78, P<.001)$; more likely to be diagnosed with dementia (5.9\% vs $3.5 \%, P<.001)$, congestive heart failure $(12.3 \%$ vs $9.9 \%, P<.001)$, and cancer $(6.1 \%$ vs $3.3 \%, P<.001)$; more likely to die during the admission ( $16.5 \%$ vs $10.9 \%, P<.001)$; and, conditional on survival to discharge, more likely to be discharged with hospice (17\% vs $3 \%, P<.001)$ than those without (Table 2).

At the hospital level, ACP rates varied from $0 \%$ to $35 \%$ (mean $5.2 \%$ ) of all admissions. In analyses restricted to physicians

TABLE 2. Characteristics of Patients over the Age of 65 Managed by a National Hospital Physician Practice Management Group During Quarter 1 2017, by Advance Care Planning Billing During the Admission

\begin{tabular}{|c|c|c|c|}
\hline Variable & $\begin{array}{c}\text { ACP billed } \\
(n=6,146)\end{array}$ & $\begin{array}{l}\text { No ACP billed } \\
(n=107,466)\end{array}$ & $P$ Value \\
\hline Age, mean (SD) & $80.26(8.82)$ & $77.71(8.44)$ & $<.001$ \\
\hline "No" to the SQ*, n (\%) & $3,414(55.56)$ & $37,862(35.24)$ & $<.001$ \\
\hline \multicolumn{4}{|l|}{ Diagnoses, n (\%) } \\
\hline Dementia & $362(5.89)$ & $3,787(3.52)$ & $<.001$ \\
\hline Congestive heart failure & $755(12.28)$ & $10,604(9.86)$ & $<.001$ \\
\hline Chronic pulmonary disease & $650(10.57)$ & $12,870(11.98)$ & .001 \\
\hline Renal failure & $7(0.11)$ & $181(0.17)$ & .306 \\
\hline Liver disease & $46(0.75)$ & $564(0.52)$ & .020 \\
\hline Metastatic cancer & $82(1.33)$ & $611(0.57)$ & $<.001$ \\
\hline Solid tumor w/o metastasis & $269(4.81)$ & $2,924(2.72)$ & $<.001$ \\
\hline \multicolumn{4}{|l|}{ Discharge status, n (\%) } \\
\hline Home & $1,973(32.10)$ & $56,174(52.27)$ & $<.001$ \\
\hline Home with home healthcare & $926(15.07)$ & $15,701(14.61)$ & .7004 \\
\hline Skilled nursing facility & $1,161(18.89)$ & $16,627(15.47)$ & .002 \\
\hline Inpatient rehabilitation facility & $172(2.80)$ & $3,431(3.19)$ & .7758 \\
\hline Long-term acute care hospital & $52(0.85)$ & $616(0.57)$ & .8006 \\
\hline Hospice & $851(13.85)$ & $3,233(3.01)$ & $<.0001$ \\
\hline Deceased & $1,011(16.45)$ & $11,684(10.87)$ & $<.0001$ \\
\hline
\end{tabular}

*SQ: "Would you be surprised if the patient died in the next year?" was only asked for 73,731 admitted, nonobservation status hospital-based encounters

Abbreviations: $\mathrm{ACP}$, advance care planning; $\mathrm{SD}$, standard deviation; $\mathrm{SQ}$, surprise question. 
seeing at least 30 patients 65 years of age and older during the quarter, physician-level ACP rates varied from $0 \%$ to $93 \%$ (mean 5.4\%). The majority of all ACP discussions were attributable to one-quarter of physicians. One-third of physicians never billed for ACP.

In a hierarchical logistic regression model accounting for observable patient characteristics and clustering at the physician and hospital level, the adjusted ACP rate for an "average" patient (age 77.85 with the most common clinical conditions) was $13.6 \%$ if the hospitalist answered "no" to the SQ, 9.6\% if the hospitalist answered "yes," and $10.1 \%$ if the hospitalist was not asked the SQ ( $P$ value of difference $<.0001$ ). From this model, we also calculated an ICC at the physician level of 0.044 and at the hospital level of 0.079. The physician level ICC corresponds to a $4.5 \%$ absolute increase in ACP when one moves from a physician at the mean to a physician $1 \mathrm{SD}$ above the mean (ie, moving $1 \mathrm{SD}$ up the scale of the latent variable underlying the random effect). The hospital level ICC corresponds to a $6.3 \%$ absolute increase in ACP when one moves from a hospital at the mean to a hospital $1 \mathrm{SD}$ above the mean. The $4.5 \%$ absolute increase in ACP due to physician practice patterns and $6.3 \%$ absolute increase in ACP due to hospital practice patterns are both greater than the estimated increase in ACP from the hospitalist answering "no" instead of "yes" to the SO (3.6\%).

\section{DISCUSSION}

In this large national hospital-based physician practice group, the rates of ACP among acute care patients 65 years of age and older were very low despite the use of education and ITand incentive-based strategies to encourage ACP conversations among seriously ill older adults. Priming physicians to reflect on the patient's risk of dying at the time of admission was associated with the doubling of ACP rates.

Despite some lawmakers' concerns that the ACP billing code may be overused and therefore become a financial burden to the Medicare program, we find the very low use of ACP billing in a population for whom having goals of care conversations is critical-seriously ill older adults who the physician would not be surprised if they died in the next year. This gap is significant because these ACP conversations, when they did occur, were associated with a comfort-focused trajectory, including a more than four-fold increase in hospice referral at discharge.

Causal inference is limited because of the observational nature of the study. While we hypothesize that priming the physicians to reflect on prognosis activated them to prioritize $A C P$, based on a prior scenario-based randomized trial, ${ }^{7}$ illness severity likely drives ACP conversations. Specifically, patients on observation status (who had missing SO data) and those for whom the physician answered "yes" to the SQ are less sick than other patients. Additional decision-making heuristics in addition to mortality risk may influence ACP conversations, as suggested by the independent influence of diagnoses, such as dementia or cancer, on ACP. Notably, however, the large amounts of unexplained variation at the physician and the hospital levels exceed the amounts explained by any individual observed patient factor.

Other key limitations of this study include the use of ACP billing as a primary outcome rather than observed and documented ACP conversations and the lack of information on the quality of ACP conversations. These findings reflect the uptake of ACP billing rates soon after the code was introduced. ACP billing rates have likely increased since the first quarter of 2017. Future work should explore diffusion and variation in physician-specific use over time. Finally, despite the nationwide sample, findings may not be generalizable to hospitalists who have not received training and financial incentives for ACP billing.

This study reinforces the possibility that variation in ACP conversations may contribute to variation in end-of-life treatment intensity between providers. ${ }^{8-10}$ Low ACP rates among even those with high hospitalist-predicted mortality risk and considerable between-provider variation underscore the need for quality improvement interventions to increase hospital-based ACP.

\section{Acknowledgments}

The authors thank Jared Wasserman, Maxwell Bessler, Devon Zoller MD, Mark Rudolph MD, Kristi Franz, and Weiping Zhou for their research assistance.

Disclosures: The authors have nothing to disclose.

Funding: National Institute on Aging award P01 AG019783

\section{References}

1. Mullick A, Martin J, Sallnow L. An introduction to advance care planning in practice. BMJ. 2013;347:f6064. doi: 10.1136/bmj.f6064.

2. Sudore RL, Lum HD, You JJ, et al. Defining advance care planning for adults: a consensus definition from a multidisciplinary Delphi panel. J Pain Symptom Manage. 2017;53(5):821-832. doi: 10.1016/j.jpainsymman.2016.12.331

3. Medicare spending and utilization for advance care planning (ACP) services in 2016. Analysis of CMS data posted by the Coalition to Transform Advanced Care https://www.thectac.org/2017/08/use-billing-codes-advancecare-planning-exceeds-projections/. Accessed February 2018.

4. Moss AH, Ganjoo J, Sharma S, et al. Utility of the "surprise" question to identify dialysis patients with high mortality. Clin J Am Soc Nephrol. 2008;3(5):1379-1384. doi: 10.2215/CJN.00940208.

5. Downar J, Goldman R, Pinto R, Englesakis M, Adhikari NK. The "surprise question" for predicting death in seriously ill patients: a systematic review and meta-analysis. CMAJ. 2017;189(13):E484-E493. doi: 10.1503/ cmaj. 160775.

6. Aleccia J. Docs bill Medicare for end-of-life advice as 'death panel' fears reemerge. Kaiser Health News, February 2017

7. Turnbull AE, Krall JR, Ruhl AP, et al. A scenario-based, randomized trial of patient values and functional prognosis on intensivist intent to discuss withdrawing life support. Crit Care Med. 2014;42(6):1455-1462. doi: 10.1097/ CCM.0000000000000227.

8. Barnato AE, Mohan D, Lane RK, et al. Advance care planning norms may contribute to hospital variation in end-of-life ICU use: a simulation study. Med Decis Making. 2014;34(4):473-484. doi: 10.1177/0272989X14522099

9. Barnato $A E$, Tate JA, Rodriguez KL, Zickmund SL, Arnold RM. Norms of decision making in the ICU: a case study of two academic medical centers at the extremes of end-of-life treatment intensity. Intensive Care Med. 2012;38(11):1886-1896. doi: 10.1007/s00134-012-2661-6.

10. Wright AA, Zhang B, Ray A, et al. Associations between end-of-life discussions, patient mental health, medical care near death, and caregiver bereavement adjustment. JAMA. 2008;300(14):1665-1673. doi: 10.1001/ jama.300.14.1665. 